\title{
Relative changes of risks on having alzheimer's dementia based on metabolism and lifestyle factors using GH-Method: math-physical medicine (No. 372)
}

\begin{abstract}
The author has been reading dementia articles for over the past two years. Recently, he read four articles about Alzheimer's dementia (AD) diseases cited in References. ${ }^{1-4}$ He was inspired by them and decided to utilize his developed GH-Method: math-physical medicine (MPM) and his collected big data of health conditions and lifestyle details to calculate his relative risk change in developing AD.
\end{abstract}

This article applies many concepts, theories, and techniques from mathematics, physics, engineering, computer science and artificial intelligence, which the author learned in his lifetime. This big data analytics project contains 9 years of health conditions and lifestyle details. The basic concept of this article is similar to his previous research work and published articles regarding various relative changes of risk on having a cardiovascular disease (CVD), stroke, chronic kidney disease (CKD), and cancer. However, this specific article focuses on metabolism and certain lifestyle factors which influence $\mathrm{AD}$.

Over the past 11 years, the author has self-studied and conducted his own medical research with his developed MPM approach, where the original focus was diabetes and its related complications. However, he soon discovered that the root cause of his diabetes and multiple complications are a result of his poor lifestyles since 1980. In 2010, when he initiated his own study and research on endocrinology, he made a vow to himself that within 10 years, he would understand his entire health situation and related medical issues using his acquired academic tools, i.e. mathematics, physics, engineering, and computer science, without biology and chemistry. He has researched all of his chronic diseases and their induced complications, including obesity, diabetes, hypertension, hyperlipidemia, CVD, CKD, foot ulcer, diabetic retinopathy, and hypothyroidism.

Eleven years later, he finally realized that the biomedical system is the most sophisticated and also complicated system he has ever dealt with. The main reason is that body cells and internal organs are living organic materials, different from his familiar inorganic engineering materials. They go through many lifecycles and stages, such as growth, sickness, damage, healing, mutation, rebirth, and death which are different from the materials he has studied, such as steel, concrete, soil, ocean water, etc. in various systems of aerospace, defense, computer, electronics and semiconductors devices, mechanical and structural engineering, financial and management systems.

During this time frame of continuous self-study and medical research, he has identified a mainstream of his thoughts and his research ways, i.e. starting from lifestyle through metabolism and immunity, and before reaching various diseases. This route has guided him to expand his interests and efforts from diabetes into many other related branches of medicine, such as dementia and cancer. For example, the numerous articles mentioned in Reference 5, as shown in Figure 3, clearly indicates dementia prevention through lifestyle management. Even cancers have approximately $45.2 \%$ cases related to various metabolic disorders and poor lifestyles. ${ }^{5}$

The above descriptions outline his philosophy, causes and efforts in producing this particular research note regarding $\mathrm{AD}$.

The author estimates $65 \%$ to $70 \%$ of deaths from various disease are either

\section{Introduction}

The author has been reading dementia articles for over the past two years.Recently, he read four articles about Alzheimer's dementia
Volume 5 Issue 6 - 2020

\author{
Gerald C Hsu \\ Medical Research Scientist, eclaireMD Foundation, USA
}

Correspondence: Gerald C Hsu, Medical Research Scientist, eclaireMD Foundation, USA, Email g.hsu@eclairemd.com

Received: December 09, 2020 | Published: December 23, 2020

directly or indirectly related to metabolic disorders which are linked to poor lifestyles in most cases. In other words, those death-causing diseases have a common root cause, which is lifestyle. Unfortunately, so many patients are "lazy" and seek for a "quick fix" through their medical doctors. In fact, there is no true "fix" or permanent "repair" for many endocrinological diseases, particularly when they occur inside the human internal organs, including the brain. Lifestyle management combined with strong willpower and persistence is the only way to deal with the root cause of many of these diseases.

The author wants to share his acquired knowledge and learned experiences with other patients in order to avoid developing many diseases or to "reduce the risks of having those death-causing diseases".

In this article, he has identified six extremely high correlation coefficients $(>90 \%)$ existing among AD risk, metabolism, and certain specific lifestyle factors. This means that the $\mathrm{AD}$ risk probability is indeed closely related to both metabolic conditions and lifestyle details as mentioned in the four referenced articles.

Furthermore, through his sensitivity analysis using two different sets of weighting factor split between metabolism index and lifestyle factors, i.e. $50 \%$ vs. $50 \%$ and $33 \%$ vs. $67 \%$, the nominal difference of $<4 \%$ risk is observed from these 2 resulting $\mathrm{AD}$ risk percentages.

He was pleased to discover that his relative changes of risk on having $\mathrm{AD}$ is continuously being reduced year over year, even though these risk percentages are only "relative figures", not "absolute numbers". This encouraging phenomenon is directly resulting from his continuous efforts on improving his metabolism through lifestyle management.

(AD) diseases cited in References. ${ }^{1-4}$ He was inspired by them and decided to utilize his developed GH-Method: math-physical medicine (MPM) and his collected big data of health conditions and lifestyle details to calculate his relative risk change in developing AD. 
This article applies many concepts, theories, and techniques from mathematics, physics, engineering, computer science and artificial intelligence, which the author learned in his lifetime. This big data analytics project contains 9 years of health conditions and lifestyle details. The basic concept of this article is similar to his previous research work and published articles regarding various relative changesof risk on having a cardiovascular disease (CVD), stroke, chronic kidney disease (CKD), and cancer. However, this specific article focuses on metabolism and certain lifestyle factors which influence AD.

\section{Methods}

\section{Background}

To learn more about the author's GH-Method: math-physical medicine (MPM) methodology, readers can refer to his article to understand his developed MPM analysis method in. ${ }^{6}$

\section{Alzheimer's dementia (AD)}

The following paragraphs in this sub-section are excerpts from References. ${ }^{1-4}$

\section{AD overall picture}

"Alzheimer's dementia is the most common form of dementia, and probably the best studied. Alzheimer disease is on the rise in the United States, and the facts are daunting.According to the Alzheimer Association's 2020 Alzheimer's Disease Facts and Figures report, approximately 5.8 million Americans age 65 and older currently have the Alzheimer dementia (AD) disease, with this number expected to triple over the next 30 years, and nearly two-thirds of those are women. It's the sixth leading cause of death in the United States-a ranking that's expected to skyrocket as the US population ages.

And it's not just an American problem. The WHO estimates there are about 50 million people across the globe living with dementia, with nearly 10 million cases being added each year (Reference 3). According to a review published in Therapeutic Advances in Chronic Disease, this number is projected to increase to 75 million by 2030, and to 135 million by 2050 .

The cost of dementia on the worldwide healthcare system is currently more than $\$ 800$ billion and is expected to mushroom to $\$ 2$ trillion by the year 2030, and that does not account for the costs associated with informal caregiving. As a matter of fact, over 16 million Americans care for patients with Alzheimer or other dementias, without pay.'

\section{AD and lifestyle}

"The following news is based on an analysis of data collected over 8 years from almost 200,000 adults aged 60 and over in the UK (Reference 4). This study was conducted by researchers from the UK (University of Exeter Medical School, University of Oxford, University College London, The Alan Turing Institute), the US (University of Michigan, Veterans Affairs Center for Clinical Management Research in Michigan), Australia (University of South Australia), and Germany (University of Hamburg, Hamburg Center for Health Economics). The study was published in the peer-reviewed Journal of American Medical Association (JAMA)

There are measures we can take individually to help combat the problem. According to the authors of the aforementioned review, almost half of all dementia cases can be attributed to a small number of modifiable lifestyle risk factors, including smoking, obesity, and physical inactivity.

The research project used volunteers completed questionnaires at the start of the study about their lifestyles, and researchers looked at their DNA to see who carried genetic variations that have been associated with increased risk of Alzheimer's - the most common type of dementia.

The researchers found that among participants who had a higher genetic risk of getting dementia, only about 11 in every 1,000 with healthier lifestyles developed the condition during follow-up, compared with about 18 in every 1,000 with unhealthy lifestyles.

Overall, however, the findings are good news. We cannot change our genetics, but this study suggests that regardless of this, changing our lifestyles could help everyone reduce their dementia risk. This large UK cohort study has suggested that a healthy lifestyle can reduce overall dementia risk, even in people with genetic risk factors for Alzheimer's disease. ${ }^{4}$ The Daily Telegraph reported that "Bad dementia genes can be overcome through healthy living, study finds". The newspaper reports that regular exercise, not smoking, drinking sensibly, and eating a healthy diet have been found to reduce risk of getting dementia even if a person has a higher genetic risk of developing the condition."

\section{The author's notes:}

Through the author's own medical research work over 30,000 hours in past 11 years, he has also discovered that a good life habits of nonsmoking, moderate or non alcohol during, avoiding illicit drugs; plus eating right food with well-balanced nutritions, persistent exercise, having sufficient and good quality of sleep, reducing all kinds of unnecessary stresses, maintaining a regular daily life routines are definitely contributing on risk reduction of having many diseases, including cardiovascular diseases, stroke, kidney problems, micro blood vessels issues, and peripheral nervous system problems. Furthermore, a long-term good lifestyle can even "repair" some damaged internal organs, with different required time-length depends on the life-span of each particular organ's cells.

\section{Data in this report:}

The author used his collected data from 2012 with different stages. Since $1 / 1 / 2012$, he recorded his daily weight, glucose, and walking steps.Since 2013, he started to collect his blood pressure and lipid (cholesterols) data. During 2014, he developed a mathematical model of metabolismwhich includes stress, sleep, daily life routines, and more. Since 5/1/2015, he started to gather his carbs/sugar intake amount and post-meal walking steps for diabetes concerns. The data collected in the earlier years was reconstructed to provide a more complete picture based on his scattered numbers.

For example, the social connection category includes the number of contacts of real (face-to-face) and virtual (online), quality of social connection, frequency of social contacts with family members, relatives, friends, colleagues, acquaintances, and certain stresses induced from social networking. One specific example is that during the COVID-19 quarantine period in 2020, virtual connection is the major form of communication between individuals. But this new and easy way of social networking could introducemore stress into people's relationship, such as stress induced from the over information sharing and online discussion of 2020 presidential election in the US. All of these factors can affect individual scores in the social and stress categories, even including sleep. 
In 2014, he developed the metabolism index model, by using topology concept of advanced mathematics and finite-element modeling techniques of structural engineering, he developed a mathematical model of metabolism index system which includes 4-categories of disease control (body outputs) and 6-categories of lifestyle management (body inputs). He started to collect his personal detailed data on $1 / 1 / 2012$. To date, he has collected more than 2 million data regarding his health and lifestyle.

For example, among these 10 categories and their associated $\sim 500$ elements, sleep category has nearly 10 elements, including sleep hours, wake up time, and deep sleep hours, while the stress category has more than 40 elements, including physical, emotional, financial, social, environmental, and others.

Finally, he further assembled those 10-categories (with $\sim 500$ detailed elements) and combined them into two new terms: the metabolism index (MI), which is a combined daily score to show the body's health situation, and general health status unit (GHSU), which is the 90-days moving average number to show the trend. He also identified a "break-even line" at $0.735(73.5 \%)$ to separate his metabolism conditions between healthy (below 0.735 ) and unhealthy (above 0.735)

For the new AD category of social network and relationship, he must establish a subjective and relative score for each year based on his memory. And, for another new AD category of mental activities, this is an easy category for him to receive higher scores due to his daily intensive and continuous medical research work during the past 11 years

\section{Results}

Figure 1 shows the raw data collected by the author since 2012 .

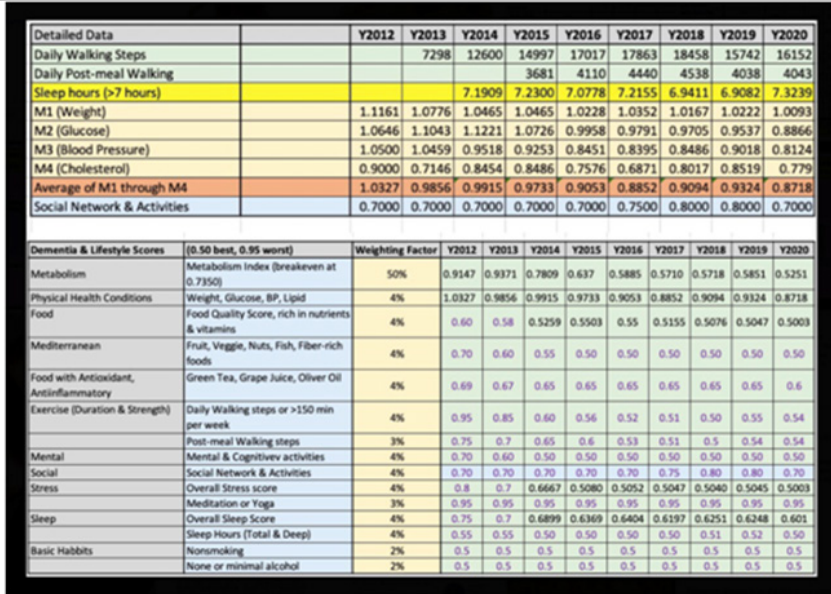

Figure I Raw input data of metabolism and Dementia factors.

The various standards used for his physical health data are:

M1: weight (170 lbs., BMI 25.0)

M2: glucose $(120 \mathrm{mg} / \mathrm{dL})$

M3: blood pressure (120/80), pulse (60) (200).

M4: triglycerides (150), HDL (40), LDL (130), total cholesterol

The input data of physical health in this study is using the linear average value of above M1 through M4
The black colored numbers are his measured data, while the purple colored numbers are his best-estimated numbers based on scattered data or memory.

The author has learned something new from this research work. He has not paid any attention to meditation or yoga since he has maintained a low stress level over the past 9 years (his daily average stress score is near the perfect level of 0.5). That is why he gives himself a score of 0.95 (1.0 is the worst score in this meditation category). However, he does understand the positive contributions from both meditation and yoga and will start practicing both in 2021 . This is a small benefit he has gained from this particular dementia study.

Figure 2 is the calculated scores with different weighting factors, i.e. weighted scores. Although the MI score with $50 \%$ weighting factors already contains 10 big categories with $\sim 500$ detailed elements, it further emphasizes the influential factors directly related to $\mathrm{AD}$, with those 14 listed contribution factors to occupy the remaining 50\% of the total weighting factors.

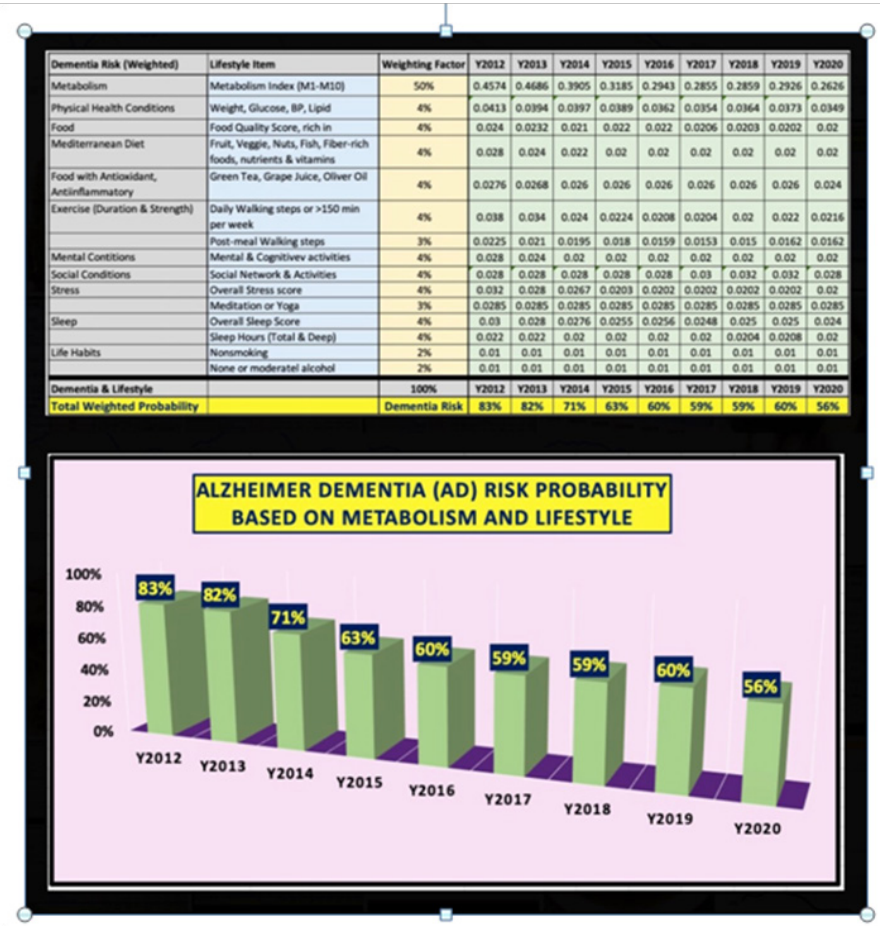

Figure 2 Weighted scores and Annual AD Risk Probability \%.

After calculating each element's weighted score in 9 different years, the summation of the MI contribution and all of the $14 \mathrm{AD}$ specific influential factors' contributions would indicate the relative changes of risk on having AD during that particular year.

The bar chart of relative changes of risk on having $\mathrm{AD}$, in the lower part of Figure 2, indicates the author'srelative changes of risk on having AD from 2012 through 2020. It should be emphasized that the risk percentage is not an "absolute risk level", but rather a "relative risk level". Therefore, the bar diagram reveals a fact that his relative changes of risk on having AD is continuously reducing towards a "less likely situation".

Figure 3 demonstrates the knowledge and information based on 115 of other people's work which are included as the reference list cited in. ${ }^{3}$ 


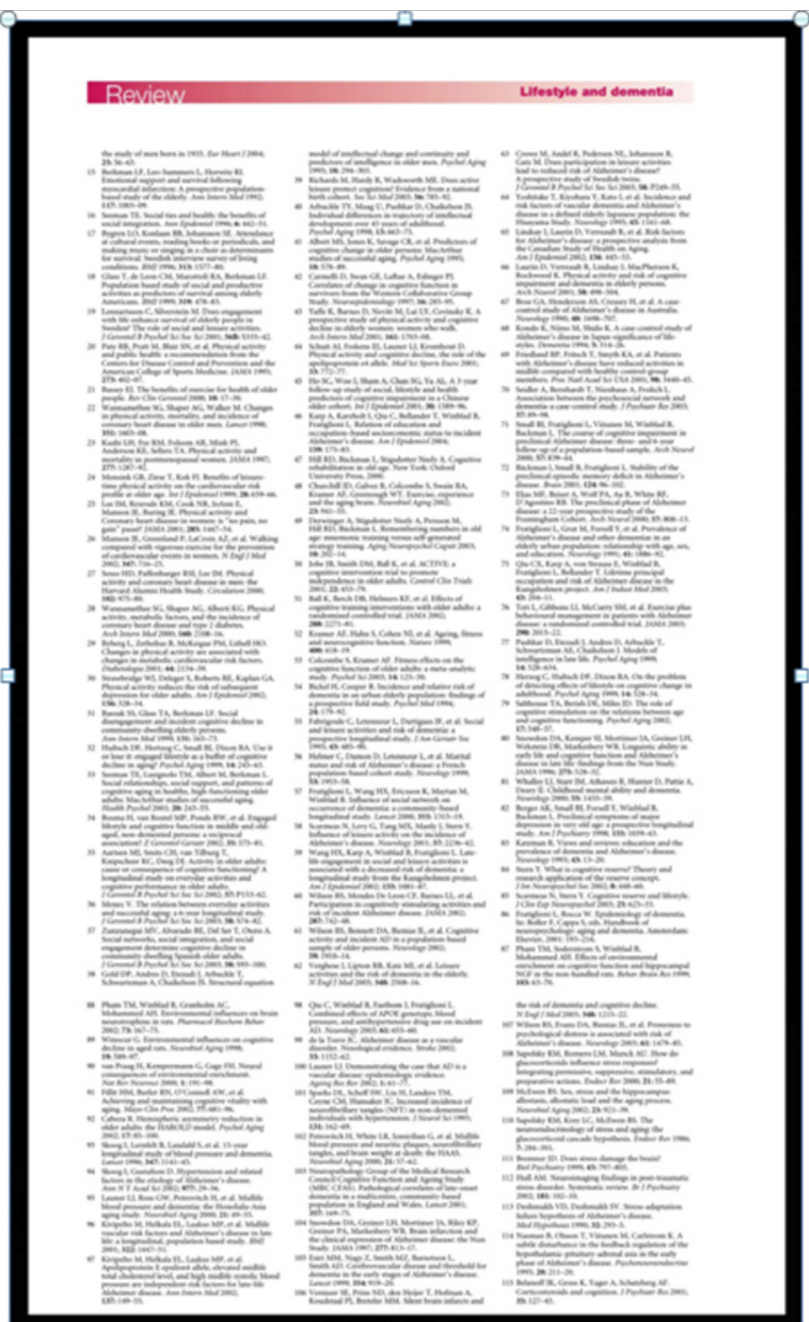

Figure 3 II 5 References in Laura Fratiglioni, The Lancet Neurology, Vol 3, June 2004, "An active and socially integrated lifestyle in late life might protect against dementia".

Figures 4 \& 5 illustrate the results of sensitivity analysis of the numerical values of $\mathrm{AD}$ risk probabilities.Figure 4 shows two different weight factors, 50/50 and 33/67 to split between MI and other $\mathrm{AD}$ factors. It also includes two cases containing six correlation coefficients calculations.

There are two key observations from Figures $4 \& 5$. First, the AD risk, metabolism, and other $\mathrm{AD}$ influential factors have extremely high correlations ( $>90 \%)$ among them. These high correlations prove that metabolism and lifestyle factors are closely related to AD. Second, the different weight distributions, e.g. $50 / 50$ vs. $33 / 67$, do not change the declining trend of $\mathrm{AD}$ risks from year to year and the numerical differences of relative changes of risk on having $\mathrm{AD}$ resulted from these two different weight splits, are within a nominal range of $-1 \%$ to $+4 \%$. These low differences of relative changes of risk on having $\mathrm{AD}(<4 \%)$ prove that once you improve your metabolism and lifestyle factors, your relative changes of risk on having $\mathrm{AD}$ will also be continuously reducing.

\section{Conclusion}

Over the past 11 years, the author has self-studied and conducted his own medical research with his developed MPM approach, where the original focus was diabetes and its related complications. However, he soon discovered that the root cause of his diabetes and multiple complications are a result of his poor lifestyles since 1980. In 2010, when he initiated his own study and research on endocrinology, he made a vow to himself that within 10 years, he would understand his entire health situation and related medical issues using his acquired academic tools, i.e. mathematics, physics, engineering, and computer science, without biology and chemistry. He has researched all of his chronic diseases and their induced complications, including obesity, diabetes, hypertension, hyperlipidemia, CVD, CKD, foot ulcer, diabetic retinopathy, and hypothyroidism.

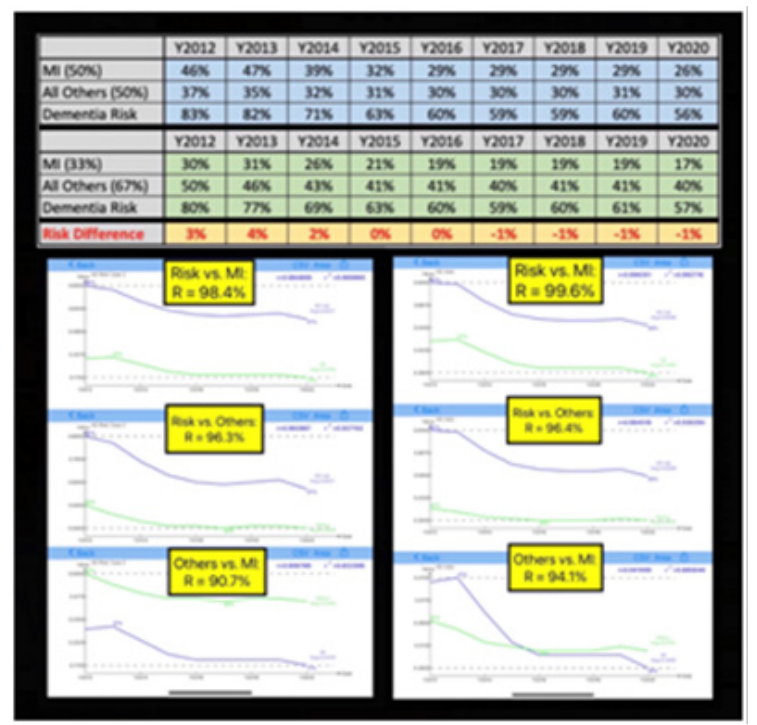

Figure 4 Sensitivity analysis of weighting factors and Correlation coefficients calculations.

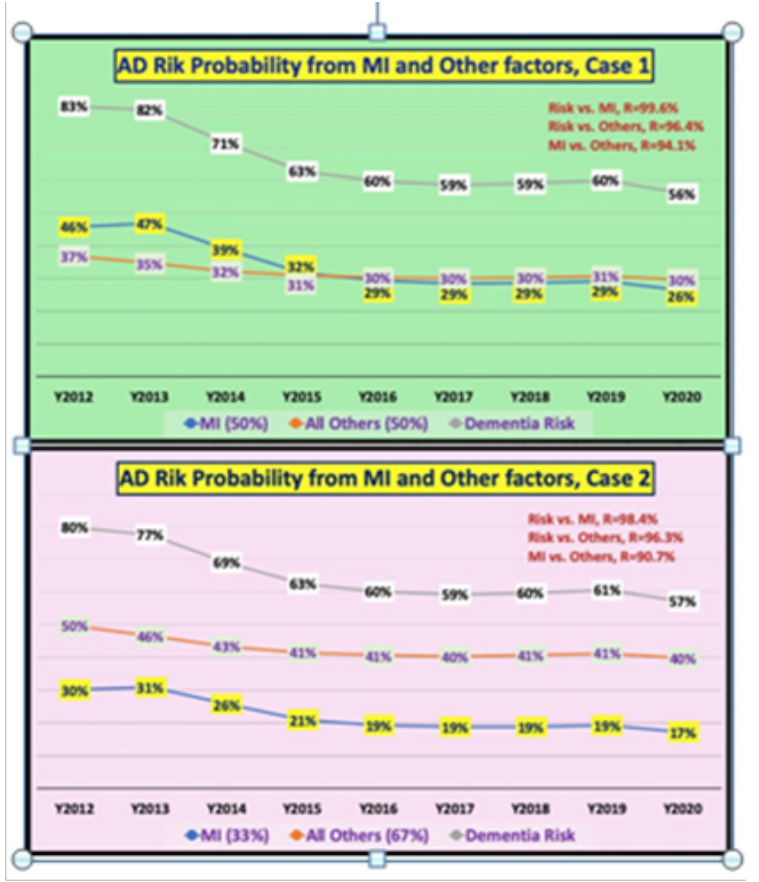

Figure 5 Comparison of $A D$ risk, $\mathrm{MI}$ and other $\mathrm{AD}$ factors for two different weighting factor splits.

Eleven years later, he finally realized that the biomedical system is the most sophisticated and also complicated system he has ever dealt 
with. The main reason is that body cells and internal organs are living organic materials, different from his familiar inorganic engineering materials. They go through many lifecycles and stages, such as growth, sickness, damage, healing, mutation, rebirth, and death which are different from the materials he has studied, such as steel, concrete, soil, ocean water, etc. in various systems of aerospace, defense, computer, electronics and semiconductors devices, mechanical and structural engineering, financial and management systems.

During this time frame of continuous self-study and medical research, he has identified a mainstream of his thoughts and his research ways, i.e. starting from lifestyle through metabolism and immunity, and before reaching various diseases. This route has guided him to expand his interests and efforts from diabetes into many other related branches of medicine, such as dementia and cancer. For example, the numerous articles mentioned in Reference 5, as shown in Figure 3, clearly indicates dementia prevention through lifestyle management. Even cancers have approximately $45.2 \%$ cases related to various metabolic disorders and poor lifestyles. ${ }^{2}$

The above descriptions outline his philosophy, causes and efforts in producing this particular research note regarding $\mathrm{AD}$.

The author estimates $65 \%$ to $70 \%$ of deaths from various disease are either directly or indirectly related to metabolic disorders which are linked to poor lifestyles in most cases. In other words, those death-causing diseases have a common root cause, which is lifestyle. Unfortunately, so many patients are "lazy" and seek for a "quick fix" through their medical doctors. In fact, there is no true "fix" or permanent "repair" for many endocrinological diseases, particularly when they occur inside the human internal organs, including the brain. Lifestyle management combined with strong willpower and persistence is the only way to deal with the root cause of many of these diseases.

The author wants to share his acquired knowledge and learned experiences with other patients in order to avoid developing many diseases or to "reduce the risks of having those death-causing diseases".

In this article, he has identified six extremely high correlation coefficients ( $>90 \%)$ existing among AD risk, metabolism, and certain specific lifestyle factors. This means that the AD risk probability is indeed closely related to both metabolic conditions and lifestyle details as mentioned in the four referenced articles.

Furthermore, through his sensitivity analysis using two different sets of weighting factor split between metabolism index and lifestyle factors, i.e. $50 \%$ vs. $50 \%$ and $33 \%$ vs. $67 \%$, the nominal difference of $<4 \%$ risk is observed from these 2 resulting AD risk percentages.
He was pleased to discover that his relative changes of risk on having $\mathrm{AD}$ is continuously being reduced year over year, even though these risk percentages are only "relative figures", not "absolute numbers". This encouraging phenomenon is directly resulting from his continuous efforts on improving his metabolism through lifestyle management.

\section{Acknowledgments}

First and foremost, the author wishes to express his sincere appreciation to a very important person in his life, Professor Norman Jones at MIT and University of Liverpool. Not only did he give him the opportunity to study for his $\mathrm{PhD}$ at MIT, but he also trained him extensively on how to solve difficult problems and conduct any basic scientific research with a big vision, pure heart, and integrity.

The author would also like to thank Professor James Andrews at the University of Iowa. He helped and supported him tremendously when he first came to the United States. He believed in him and prepared him to build his solid engineering and computer science foundation. $\mathrm{He}$ is forever grateful to his mentor, who has a kind heart and guided him during his undergraduate and master's degree work at Iowa.

\section{Conflicts of interest}

The authors declare have no conflict of interest about the publication of this paper.

\section{References}

1. Alistair Gardiner, November 24, 2020, MDLinx: "Alzheimer disease: Simple ways to prevent cognitive decline".

2. Alison Bruner, communication officer, World Health Organization, 14 May 2019 News release, Geneva: "New WHO Guidelines recommend specific interventions for reducing the risk of cognitive decline and dementia: Adopting a healthy lifestyle helps reduce the risk of dementia".

3. Bazian, Edited by NHS Website, 15 July 2019: "Healthy lifestyle reduces dementia risk even in those with genetic risk factors".

4. Laura Fratiglioni, Stephanie paillard-borg, and bengt winblad, The Lancet Neurology. http://neurology.thelancet.com: "An active and socially integrated lifestyle in late life might protect against dementia. 2004;(3),

5. Hsu Gerald C. Cancer prevention through improvements on lifestyle and metabolism using GH-Method: math-physical medicine". eclaireMD Foundation, USA, No. 342.

6. Hsu Gerald C. "Biomedical research methodology based on GHMethod: math-physical medicine". eclaireMD Foundation, USA, No. 310 